\title{
JOVIAN RADIO EMISSIONS MODELING AND THEIR FUTURE INVESTIGATION WITH EJSM
}

\author{
B. Cecconi*, S. Hess ${ }^{\dagger}$, and P. Zarka*
}

\begin{abstract}
Dynamic spectra of planetary radio emissions depend on physical and geometrical conditions: emission process; energy of emitting electrons; angle between the source magnetic field and the wave direction, which varies with frequency; location of the observer... Their modeling is an intrinsically 3-dimensional problem for which a code has been developed: SERPE / ExPRES (Simulateur d'Émissions Radio Planétaires et Exoplanétaires / Exoplanetary and Planetary Radio Emissions Simulator). This tool has been successfully applied to the modeling of arc-shaped radio emissions generated by the Io-Jupiter electro-dynamic interaction, as well as of Saturn's Kilometric Radiation dynamic spectra. It allowed us to determine the energy of the emitting electrons, to identify the important of the oblique mode in the rarefied auroral plasmas of Jupiter and Saturn and to clarify the link between the Io-Jupiter radio emissions and of the UV spot at the magnetic footprint of Io. In light of these results, we will briefly review the characteristics of radio emissions related to Jupiter's aurorae and to satellite-Jupiter interactions.

The EJSM (Europa Jupiter System Mission) mission is a unique opportunity to study the Jovian magnetosphere (from the close environment of Galilean satellites and their coupling with the Jovian magnetic field, to the auroral regions of Jupiter). It should carry the first goniopolarimetric radio receiver in Jovian orbit. We will illustrate with Cassini results the scientific enhancement of radio astronomy science brought by such capabilities that will not be available to any other mission around Jupiter. We will show how our present knowledge of Jupiter's decameter radio emissions may allow us to optimize the scheduling of low-frequency radar observations. Finally, we will discuss the unique opportunities that would be offered by multi-spacecraft magnetospheric observations in the Jovian system.
\end{abstract}

* LESIA, Observatoire de Paris, UMR CNRS 8109, 92195 Meudon, France

† Laboratory for Atmospheric and Space Physics, University of Colorado, Boulder, CO 80303-7814, USA 
